\title{
Reversible magnetoelectric switching in multiferroic three-dimensional nanocup heterostructure films
}

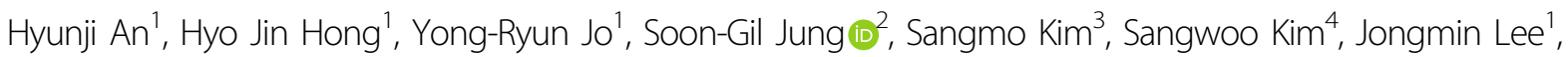 \\ Hojoong Choi ${ }^{1}$, Hongji Yoon', So-Young Kim', Jaesun Song', Sang Yun Jeong ${ }^{1}$, Byoung Hun Lee', Tae-Yeong Koo ${ }^{4}$,

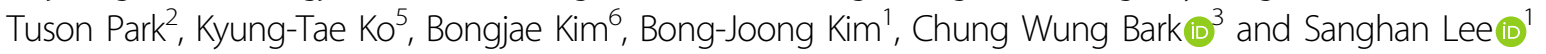

\begin{abstract}
Self-assembled nanocomposite films containing ferroelectric and ferromagnetic phases have attracted enormous research interest because they are the most promising candidates for practical multiferroic applications. However, obtaining a genuine magnetoelectric (ME) coupling effect is still challenging in this research area. To substantially improve the ME effect, new heterostructure designs with efficient strain control between two phases are urgently needed. Herein, a novel three-dimensional (3D) nanocup architecture of a heterostructure film is developed. To establish the unique architecture, a heavily $\mathrm{Co}$, Fe-doped ferroelectric $\mathrm{Bi}_{3.25} \mathrm{La}_{0.75} \mathrm{Ti}_{3} \mathrm{O}_{12}$ (BLT) target was used during the growth of BLT thin films via pulsed laser deposition. Consequently, 3D nanocup-structured $\mathrm{CoFe}_{2} \mathrm{O}_{4}(\mathrm{CFO})$ particles formed inside the BLT via spontaneous nucleation and agglomeration. The 3D nanocup BLT-CFO film exhibited magnetically controlled reversible dielectric switching, which is direct evidence of strong ME coupling caused by the efficient interfacial strain coupling and low leakage of the novel nanocup architecture. The obtained results strongly suggest that the 3D nanocup heterostructure film significantly improves the ME coupling effect. In addition, we propose a new paradigm in the architecture design of self-assembled nanocomposite films for diverse multifunctional devices.
\end{abstract}

\section{Introduction}

Over the past decades, the ability to synthesize selfassembled nanocomposite films of complex oxides has paved the way for creating new physical phenomena ${ }^{1-5}$. Until now, these self-assembled nanocomposite films have demonstrated a variety of fascinating physical phenomena, including enhanced flux pinning in high-temperature superconductors ${ }^{6,7}$, strain-enhanced ferroelectricity and multiferroics $^{8,9}$, enhanced ferromagnetism ${ }^{10}$,

Correspondence: Bong-Joong Kim (kimbj@gist.ac.kr) or Chung

Wung Bark (bark@gachon.ac.kr) or Sanghan Lee (sanghan@gist.ac.kr)

${ }^{1}$ School of Materials Science and Engineering, Gwangju Institute of Science

and Technology, Gwangju 61005, Republic of Korea

${ }^{2}$ Center for Quantum Materials and Superconductivity (CQMS), Department of

Physics, Sungkyunkwan University, Suwon 16419, Republic of Korea

Full list of author information is available at the end of the article.

These authors contributed equally: Hyunji An, Hyo Jin Hong magnetoresistance ${ }^{11}$, novel electronic/ionic transport ${ }^{12}$, and coupling of dielectric and optical effects ${ }^{13}$. Furthermore, many other areas remain to be explored for potential practical applications in various materials systems.

Self-assembled nanocomposite films containing ferroelectric and ferromagnetic phases have attracted considerable attention because they can be used in applications such as high-density memories, highly sensitive magnetic sensors, and electrically tunable spintronics $^{14,15}$. Despite the possible applicability to various ME devices, a single-phase multiferroic rarely exists in nature or has weak multiferroism at room temperature ${ }^{14}$. Hence, many researchers have attempted to overcome this problem with a variety of approaches, which include substituting magnetic impurities or cations and making

\section{(c) The Author(s) 2019}

(c) Open Access This article is licensed under a Creative Commons Attribution 4.0 International License, which permits use, sharing, adaptation, distribution and reproduction cc) in any medium or format, as long as you give appropriate credit to the original author(s) and the source, provide a link to the Creative Commons license, and indicate if changes were made. The images or other third party material in this article are included in the article's Creative Commons license, unless indicated otherwise in a credit line to the material. If material is not included in the article's Creative Commons license and your intended use is not permitted by statutory regulation or exceeds the permitted use, you will need to obtain permission directly from the copyright holder. To view a copy of this license, visit http://creativecommons.org/licenses/by/4.0/. 
heterostructure materials based on ferroelectric and ferromagnetic materials ${ }^{16,17}$. In single-phase multiferroics, barium hexaferrites have attracted attention because of their interesting magnetic structure, in which electric polarization can be induced at room temperature, and their high resistivity. By controlling the compositional ratio and substituted divalent ions in hexaferrite, various crystal structures have been found; consequently, the room-temperature magnetoelectric effect ${ }^{18}$ and the converse magnetoelectric effect ${ }^{19}$ were realized for the first time in a single multiferroic material. Since the discovery of self-assembled $\mathrm{BaTiO}_{3}-\mathrm{CoFe}_{2} \mathrm{O}_{4}$ films with strong multiferroism at room temperature ${ }^{8}$, many studies on such multiferroic heterostructure films have been conducted $^{14,20-23}$. However, it is still challenging to investigate an intrinsic ME coupling effect by eliminating other artifacts due to the structural limitation of conventional heterostructure films ${ }^{8,24,25}$. Therefore, new heterostructure designs are urgently required to obtain a reliable ME effect ${ }^{14,15}$.

Figures S1a-c (Supplementary Information) show schematics of the most common architectures of multiferroic heterostructure films: 0-3 (particulate heterostructure), 2-2 (layered heterostructure), and 1-3 (vertically aligned nanocomposite, VAN) ${ }^{20,21}$. Among these, 1-3 VAN films exhibit a relatively large ME effect owing to the reduced clamping effect of the substrate; in addition, efficient strain coupling is achieved via the larger interfacial area with intrinsic three-dimensional (3D) heteroepitaxy ${ }^{14}$. However, unlike the $0-3$ and 2-2 types, 1-3 VAN films are susceptible to leakage, which results from the low resistance of the magnetic pillars penetrating the film matrix. Eventually, efficient epitaxial strain control between ferroelectric and ferromagnetic phases through both large interfacial areas and excellent interfacial lattice coherence may substantially enhance the ME coupling effect; thus, a reliable ME effect can be achieved by overcoming the leakage issue. However, various recent heterostructures, such as the $\mathrm{NiFe}_{2} \mathrm{O}_{4}-\mathrm{CoFe}_{2} \mathrm{O}_{4}$ mixed phase $^{26,27}$, well-ordered nanodot arrays ${ }^{28,29}$, and quasi (0-3) nanocomposites ${ }^{25}$, cannot fulfill the requirements specified above. Furthermore, these structures require complicated fabrication processes, which are not suitable for potential practical applications. Hence, a new architecture for multiferroic heterostructure films should be developed via simple and innovative approaches.

In this study, we propose a newly designed 3D nanocup heterostructure film (Fig. S1d, Supplementary Information) that exhibits reversible and genuine ME switching. To fabricate such a unique 3D nanocup architecture, we used a Co, Fe-incorporated ferroelectric $\mathrm{Bi}_{3.25} \mathrm{La}_{0.75} \mathrm{Ti}_{3} \mathrm{O}_{12}$ (BLT) ceramic target. During pulsed laser deposition (PLD), 3D nanocup-structured $\mathrm{CoFe}_{2} \mathrm{O}_{4}$ (CFO) particles were formed by spontaneous nucleation and agglomeration inside the BLT film. Because of the formation of ferromagnetic CFO nanocups inside the ferroelectric BLT matrix, the obtained heterostructure film exhibited room-temperature multiferroism. Furthermore, the 3D nanocup BLT-CFO film showed magnetically controlled reversible dielectric switching originating from its structural characteristics, namely, the large interfacial area, excellent interfacial lattice coherence, and low leakage.

\section{Materials and methods \\ Density functional calculations}

Electronic structure calculations were performed using the projector-augmented-wave method implemented in the Vienna ab initio simulation package ${ }^{30,31}$. The method is explained in detail in our previous study ${ }^{32}$. For the simulation of the substrate strain, we varied the in-plane lattice parameter of $\mathrm{SrTiO}_{3}$ by up to $4 \%$, considering both tensile and compressive strains. Full atomic relaxation was performed for all studied ranges at the corresponding inplane lattice parameter. The most likely Co and Fe sites in the incorporated BLT were identified by comparing all possible sites and combinations. The most stable configuration was used for the energetics comparison shown in Fig. 1a.

\section{Sample preparation}

The Co, Fe-incorporated BLT ceramic was synthesized via a solid-state reaction. The chemical concentrations were precisely controlled to obtain $\mathrm{Bi}_{3.25} \mathrm{La}_{0.75} \mathrm{Co}_{0.5} \mathrm{Fe}_{0.5^{-}}$ $\mathrm{Ti}_{2} \mathrm{O}_{12}$. The ceramic target had a pristine BLT phase, as indicated by the XRD $\theta-2 \theta$ results $^{33,34}$. 3D BLT-CFO films with a thickness of $10-180 \mathrm{~nm}$ were fabricated on $\mathrm{SrTiO}_{3}(001)$ substrates at a high temperature of $850{ }^{\circ} \mathrm{C}$, oxygen pressure of $100 \mathrm{mTorr}$, and laser fluence of $2 \mathrm{~J} /$ $\mathrm{cm}^{2}$ using PLD (KrF, $\lambda=248 \mathrm{~nm}$ ).

\section{Structural characterizations}

The structural properties were analyzed via XRD (D8 Advance, Bruker) using $\mathrm{Cu} \mathrm{K} \alpha$ radiation. RSM was performed using the $3 \mathrm{~A}$ beamline at the Pohang Light Source. Surface morphologies were observed using a fieldemission scanning electron microscope (Hitachi S-4700) and atomic force microscope (Park Systems NX10). The structural characteristics and elemental mapping were investigated using a high-resolution transmission electron microscope (TecnaiTM G2 F30 S-Twin, $300 \mathrm{KeV}, \mathrm{FEI}$ ).

\section{Measurements of physical properties}

The MFM image was measured with a magnetic coating tip using a magnetic force microscope (PSIA, XE-100). After the topographic profile was obtained, the MFM measurement was performed at a lift height of $50 \mathrm{~nm}$ with a magnetic tip. The magnetic properties 

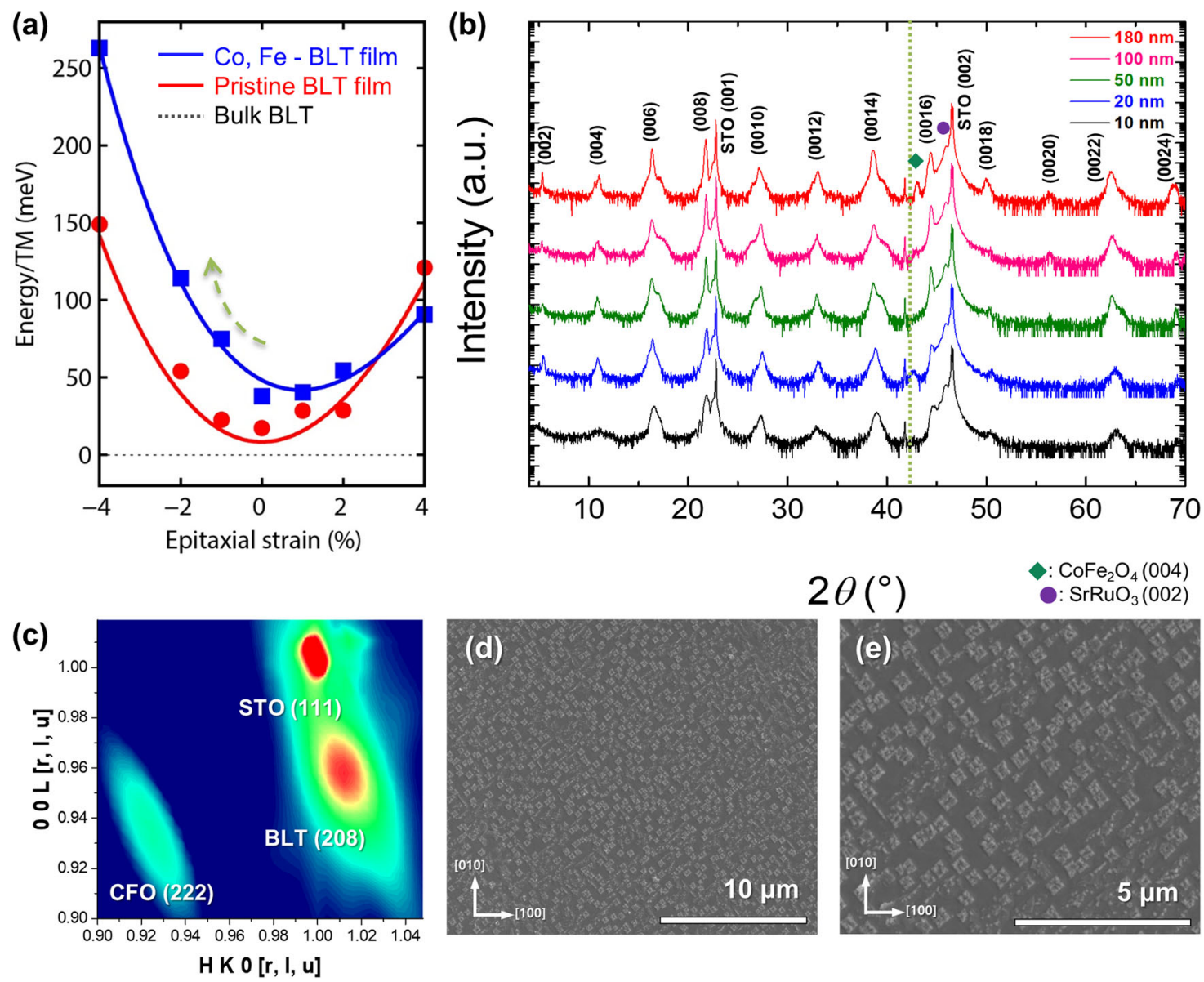

Fig. 1 Emergence of BLT-CFO heterostructure films. a DFT calculations for both pristine BLT and Co, Fe-incorporated BLT films. The green arrow indicates the strain relaxation of the films with increasing thickness. $\mathbf{b}$ XRD $\theta-2 \theta$ patterns of BLT-CFO heterostructure films with thicknesses of 10-180 $\mathrm{nm}$. (Here, the BLT phases are represented by a number, and the SRO and CFO phases are represented by a symbol to easily distinguish each phase.) The green dotted line shows the strain relaxation of CFO (004). c RSM data for the 180-nm-thick film. $\mathbf{d}$, e SEM images of the 180-nm-thick film. e Magnified image of $\mathbf{d}$.

were characterized using an MPMS (Quantum Design $5 \mathrm{~T}$ ). For electrical measurements, Pt electrodes were deposited with a thickness of $\sim 100 \mathrm{~nm}$ on all samples using an RF sputter. The electrodes have a circular shape with a diameter of $140-150 \mu \mathrm{m}$, and the number of CFO nanocups was calculated to be approximately 36000 ea per top electrode based on the SEM image. The $P-E$ loops were measured using a ferroelectric analyzer (Precision Multiferroic, Radiant Technologies, Inc.). For the measurement, the BLT and 3D BLT-CFO films (both $270 \mathrm{~nm}$ thickness) were fabricated on STO (001) and STO (110) substrates covered with $\mathrm{SrRuO}_{3}$ (SRO). The dielectric constants were measured using an LCR Meter (Keysight, E4980A). For the MD measurement, the dielectric constants were measured for the BLT and 3D BLT-CFO films (both $270 \mathrm{~nm}$ thickness) using an LCR Meter (Agilent, 4284A) under a magnetic field (PPMS, Quantum Design $9 \mathrm{~T}$ ).

\section{Results and discussion}

\section{Emergence of the BLT-CFO heterostructure film}

Ferroelectric BLT, i.e., La-doped $\mathrm{Bi}_{4} \mathrm{Ti}_{3} \mathrm{O}_{12}$ (BiT), is a well-known material used for the implementation of nonvolatile ferroelectric random access memory. BLT has robust polarization and virtual resistance to polarization fatigue $^{35}$. Nevertheless, BLT self-assembled nanocomposite films have not yet been reported. Previous attempts to fabricate a self-assembled BiT-CFO nanocomposite film resulted in a $\mathrm{Bi}_{5} \mathrm{Ti}_{3} \mathrm{FeO}_{15}$ (BTFO) phase despite the use of a conventional BiT-CFO mixed target ${ }^{24}$; such a phase was obtained because of the easily tunable characteristics of the layered perovskite structure of BiT. We also confirmed that a self-assembled BTFO-CFO film can be formed via the methods shown in Fig. S2a (Supplementary Information). To fabricate a BLT-CFO heterostructure film, we devised an entirely new approach, which resembles exsolution trends and is dissimilar to conventional 
approaches. Exsolution of transition metals was proposed for designing functional catalyst nanoparticle-supported perovskite materials ${ }^{35,36}$. In particular, exsolution of B-site dopants on layered perovskite $\operatorname{PrBaMn}_{1.7} \mathrm{~T}_{0.3} \mathrm{O}_{5+\delta}(\mathrm{T}=$ $\mathrm{Mn}, \mathrm{Co}, \mathrm{Ni}$, and $\mathrm{Fe}$ ) has recently been verified ${ }^{37,38}$. Based on these studies, we grew BLT with heavy codoping of Co and $\mathrm{Fe}$ on a $\mathrm{SrTiO}_{3}$ (STO) substrate, which led to spontaneous formation of the CFO phase inside the BLT matrix $^{39}$. This novel approach allowed us to obtain a 3D nanocup BLT-CFO heterostructure film.

First, we studied the stability of pure and $\mathrm{Co}, \mathrm{Fe}$ incorporated BLT film phases via density functional theory (DFT) calculations. In Fig. 1a, the energy differences between the bulk BLT and film phases on STO are examined. As shown in this figure, fully strained films on STO (in-plane lattice misfit: $+1.7 \%$ ) are located at 0 points; when the film thickness increased, the epitaxial strain decreased to a negative value because of strain relaxation. The $\mathrm{Co}, \mathrm{Fe}$-incorporated film showed more instability than the pure film, as indicated by the energy difference with respect to the bulk phase (38 and $17 \mathrm{meV}$ per transition-metal atom at 0 points of epitaxial strain, respectively). Furthermore, we found that the energetics of the $\mathrm{Co}, \mathrm{Fe}$-incorporated BLT phase are highly susceptible to the epitaxial strain with STO (001), which makes the codoped phase even more unstable against the misfit strain from the substrate. According to this energetics argument, we can conclude that the $\mathrm{Co}$, Feincorporated BLT phase is highly unstable and susceptible to a secondary phase, which is demonstrated by the separation of the BLT and CFO phases.

As shown in Fig. 1b, we successfully fabricated BLTCFO heterostructure films of various thicknesses ranging from 10 to $180 \mathrm{~nm}$ on STO substrates from a Co, Feincorporated BLT target using PLD. The X-ray diffraction (XRD) $\theta-2 \theta$ patterns reveal that both the BLT and CFO phases were highly $(00 l)$-oriented in all the samples. The CFO phase, which was not observed in the ultrathin sample, was found in the samples with thicknesses greater than or equal to $20 \mathrm{~nm}$ with the gradual relaxation of the epitaxial strain following the green dotted line in Fig. 1b. The phase separation of BLT and CFO was verified for the 180-nm film through reciprocal space mapping (RSM), as shown in Fig. 1c. The XRD results show that the orientation relationships of BLT and CFO to the STO substrate are as follows: BLT[110]//STO[100]; CFO[100]//STO [100]. The in-plane and out-of-plane lattice misfits between BLT (orthorhombic, $a=5.41 \AA$, $b=5.45 \AA$, $c=$ $32.83 \AA$ ) and CFO (cubic, $a=8.40 \AA$ ) are $2.30 \%$ and $8.92 \%$, respectively. Despite the large lattice misfits, the two phases were self-assembled by the intrinsic similarities in crystal chemistry of the perovskite and spinel phases $^{8}$. We directly identified the uniformly dispersed nanocomposite film via SEM (see Fig. 1d, e). We emphasize that the BLT-CFO nanocomposite film can be realized without phase transformation of the pristine ferroelectric phase by utilizing a magnetic cationincorporated target (Fig. S2b, Supplementary Information).

\section{Spontaneous nucleation and agglomeration}

To investigate the growth mechanism of the nanocomposite films, atomic force microscopy (AFM) measurements were performed on 10-, 20-, 50-, and 180-nm films (Fig. 2a-d). Small CFO nanoparticles nucleated in the initial stage. As the thickness increased, larger CFO nanoparticles formed via the agglomeration of CFO nuclei. The results confirm that the CFO nanoparticles uniformly formed through spontaneous nucleation and agglomeration (see Fig. 2d). In Fig. 2e, the noncontact mode AFM topography for the $180-\mathrm{nm}$ film is shown. After obtaining a topographic profile, we measured the magnetic force microscopy (MFM) amplitude and phase with a magnetic coating tip (Fig. 2f, g). The MFM amplitude and phase images show that the magnetic signals were detected only on the CFO nanoparticles and not on the BLT matrix.

In conventional VAN films, CFO is generally formed as nanopillars with a width of 20 to $200 \mathrm{~nm}$; for example, CFO nanopillars in the $\mathrm{BaTiO}_{3}$ matrix have circular shapes with diameters of $20-30 \mathrm{~nm}^{8}$, and those in the $\mathrm{BiFeO}_{3}$ matrix have square shapes with widths of $50-200 \mathrm{~nm}^{40}$. The difference in shapes is caused by the different binding energies between various perovskite ferroelectrics and CFO. However, in this study, large nanoparticles (width: $500 \mathrm{~nm}-1 \mu \mathrm{m}$ ) were formed by the aggregation of CFO nuclei with small widths $(\sim 20 \mathrm{~nm})$ rather than CFO nanopillars (Fig. 2f). The growth mechanism of such nanoparticles can be represented by three main stages: (i) nucleation, (ii) coalescence, and (iii) aging. These stages constitute the general process of nucleation ${ }^{41}$. According to the general nucleation theory, the shape and size of nucleated clusters are strongly affected by the surface. Conventional CFO nanopillars are grown on the STO substrate using a two-phase separated target, whereas our CFO nanocups were grown on the BLT matrix via spontaneous nucleation. In other words, these CFO nanoparticles grew in combination with BLT on a BLT surface; thus, they have different surface and binding energies than the nanopillars formed in VAN films. As a result, these energy differences enable formation of the unique architecture of CFO in a BLT-CFO heterostructure film. The TEM data in Fig. 3 support this claim by identifying the exact structure of the CFO. These results indicate that the $\mathrm{CFO}$ nanoparticles obtained through spontaneous nucleation have a different morphology and growth mechanism than the conventional VAN films. 


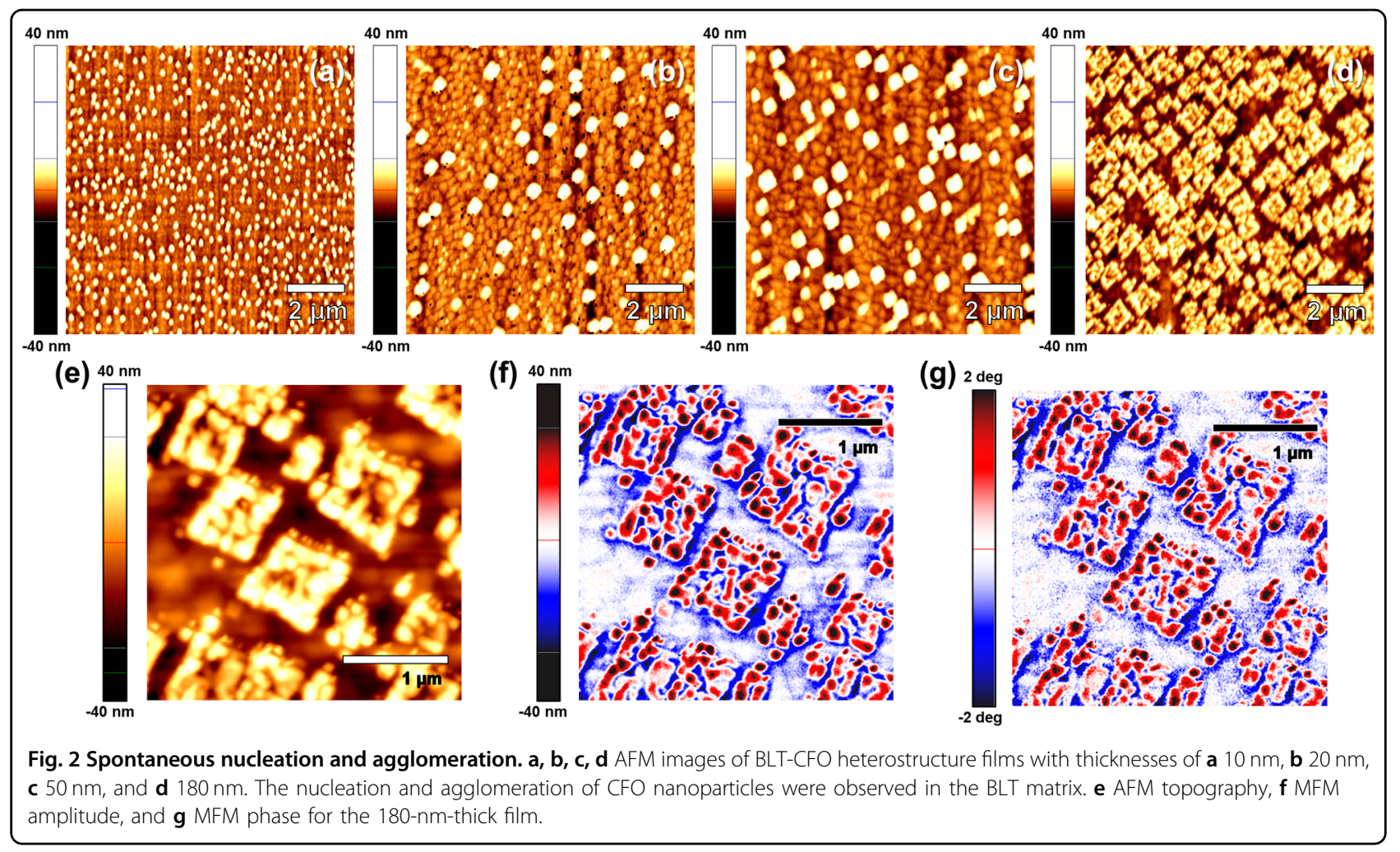

\section{D nanocup-structured nanocomposites}

For a detailed investigation, we performed a crosssectional transmission electron microscopy (TEM) analysis. The results are shown in Fig. 3a-d. The cross-sectional sample of the CFO composite was cut across the center of the structure using a focused ion beam, as depicted in Fig. 3e. The bright-field (BF) TEM images in Fig. 3a, b indicate that the nanocomposites consistently formed $\sim 10 \mathrm{~nm}$ above the STO substrate. To examine the area where the three materials were closely located, which is marked by the box in Fig. 3a, we obtained a high-resolution TEM (HRTEM) image with the corresponding fast Fourier transform (FFT) patterns for each material, as shown in Fig. 3c. These results establish that the crystal structures of STO, BLT, and CFO were simple cubic (lattice parameter: $a=3.91 \AA$ ), pseudotetragonal $(a=b=5.45 \AA, c=32.83 \AA)$, and cubic $(a=$ $8.40 \AA$ ), respectively. The diffraction pattern acquired from the area including the three materials of Fig. 3a is shown in Fig. 3d, revealing that BLT and CFO were single-crystal materials with no observable defects; they were grown epitaxially with the STO substrate, which is reflected by the parallel diffraction spots of the three materials to [002], [111], and [220] with respect to the transmitted beam. This epitaxial BLT-CFO heterostructure film had excellent coherence at both the in-plane and out-of-plane interfaces, as shown in Fig. S3 (Supplementary Information), regardless of the large in-plane (2.30\%) and out-of-plane (8.92\%) misfit strains.
To estimate the shape of the CFO particles, we characterized the structure using HRTEM and high-angle annular dark field scanning TEM (STEM), as shown in Fig. 3f, along with energy-dispersive X-ray spectroscopy (EDS). The HRTEM image with the FFT pattern obtained from the area specified by the circle on the composite in Fig. 3a shows that the material is tetragonal BLT, which indicates that the inside of the composite is filled with BLT. The EDS line scan (Fig. $3 \mathrm{~g}$ along the line marked in the STEM image in Fig. 3h shows that the inside of the composite contains significantly lower concentrations of $\mathrm{Co}$ and $\mathrm{Fe}$ than the edge, confirming that the inside comprises BLT. The EDS map in Fig. 3h identifies the extent of the BLT area (see the Co and Fe maps), indicating that the shape of the CFO particles resembles a nanosized cup with a wall thickness of $20-30 \mathrm{~nm}$.

\section{Ferroelectric and ferromagnetic properties}

The multiferroic property of the 3D nanocup BLT-CFO heterostructure film was characterized at room temperature. To confirm the ferroelectricity of the nanocomposite film, we fabricated (117)-oriented films on STO (110) covered with $\mathrm{SrRuO}_{3}$ bottom electrodes, as shown in Fig. S4a and b (Supplementary Information). It was particularly difficult to analyze the ferroelectricity of a $(00 l)$ oriented film because the primary polar axis of BLT lies along the in-plane direction ${ }^{42,43}$. The polarization of a 

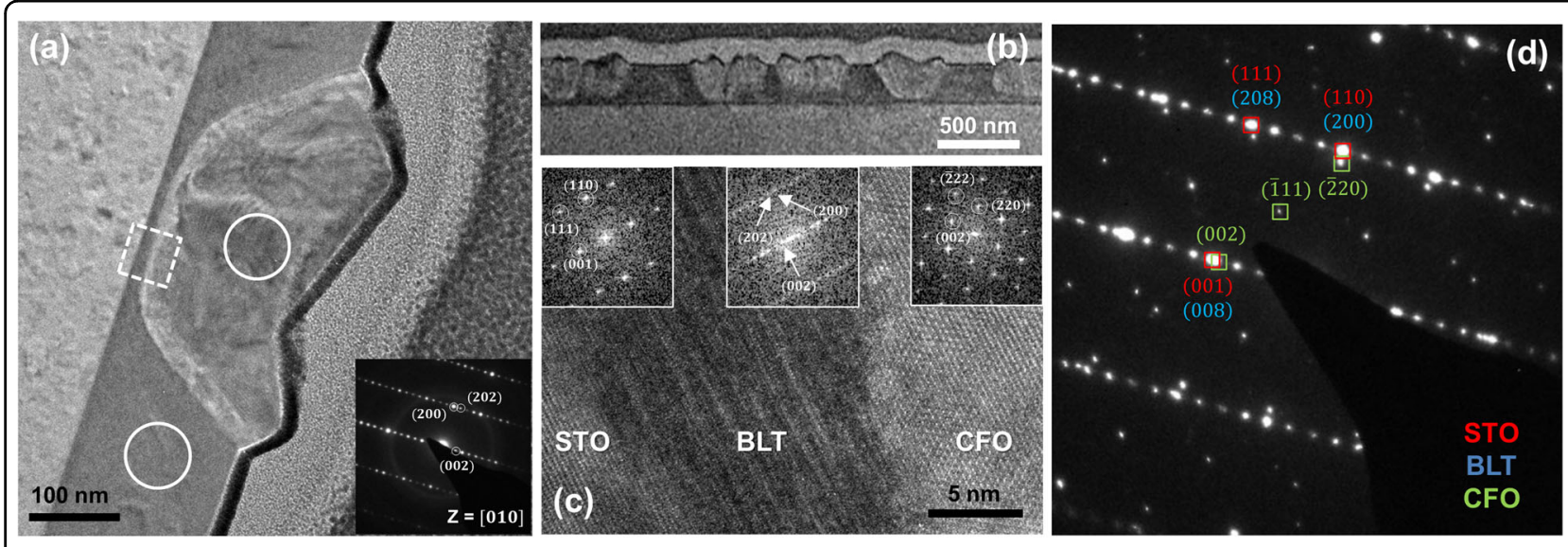

(e)

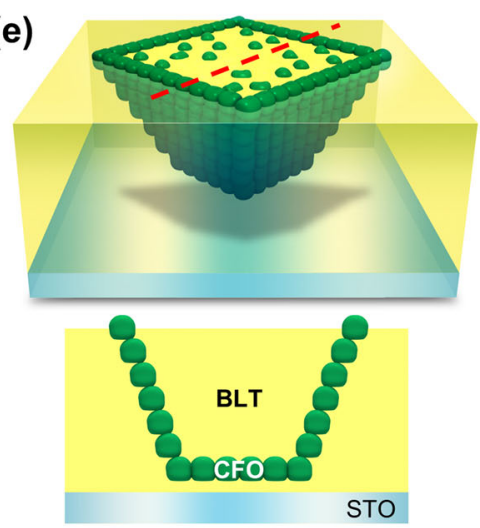

(f)

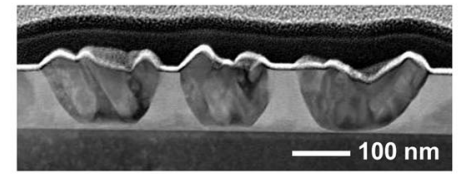

(g)

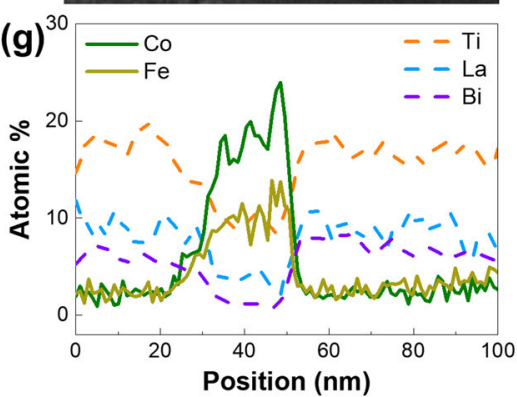

(h)
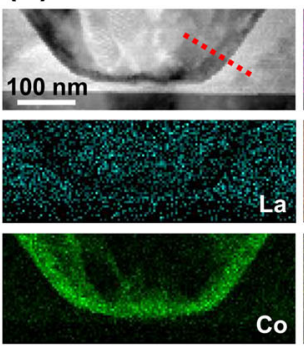

So
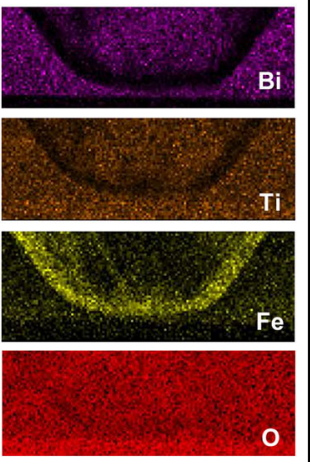

Fig. 3 Structural and compositional analyses of nanocomposites. a HRTEM image of the nanocomposite film on the STO substrate with the diffraction pattern for BLT. b Broad-view BF image of the nanocomposite film on STO. c HRTEM image of the boxed area in Fig. 3a with the FFT of each layer. $\mathbf{d}$ Diffraction pattern of the area in Fig. 3a. e Schematics of the 3D BLT-CFO nanocomposite film. $\mathbf{f}$ STEM image of the nanocomposite film on the STO substrate. $\mathbf{g}$ EDS line scan data following the line marked in the STEM image in Fig. 3h. $\mathbf{h}$ STEM image of a nanocomposite film with the EDS maps for each element.

(117)-oriented film is tilted by $60.7^{\circ}$ from the normal of the film plane. As shown in Fig. 4a, ferroelectric hysteresis loops were maintained at various frequencies. The hysteresis loop becomes depressed with a low remnant polarization compared with that of pristine BLT, which can be explained by the dilution effect based on the incorporation of paraelectric $\mathrm{CFO}^{14}$. Nevertheless, as shown in Fig. S4c and d (Supplementary Information), dielectric switching for the BLT and 3D BLT-CFO films was observed depending on the positive or negative sign of the electric field; furthermore, the value of permittivity $\left(\varepsilon_{r}: \sim 270\right)$ was similar to the reported value of BLT, while the dielectric loss $(\tan \delta)$ was $<0.1$. The low dielectric loss was also identified in the (001)-oriented 3D BLT-CFO film, as shown in Fig. 4b. We believe that the low dielectric loss may be due to the disconnect between magnetic CFO nanocups and the bottom electrode. Therefore, CFO does not act as a conducting path, and only the electric charges flowing through the BLT matrix are measured (Fig. S2d, Supplementary Information). These results demonstrate the robustness of the ferroelectric property of the 3D nanocup BLT-CFO films and indicate that there might be less leakage when such a film is applied in a device. The magnetic properties were analyzed using a magnetic-property measurement system (MPMS). Figure 4c shows that the 3D nanocup heterostructure film had strong out-of-plane ferromagnetism $\left(M_{s}: 140 \mathrm{emu} / \mathrm{cm}^{3}, M_{r}: 110 \mathrm{emu} / \mathrm{cm}^{3}, H_{c}: 0.04 \mathrm{~T}\right)$ at room temperature under a magnetic field ranging from -1 to $1 \mathrm{~T}$. The magnetization values were normalized to the volume fraction of CFO, i.e., $27 \%$ from the surface images $^{8}$. Additionally, the temperature-dependent magnetization reveals that the 3D nanocup heterostructure film had stronger in-plane ferromagnetic properties than the paramagnetic BLT film shown in Fig. 4d. These results directly demonstrate the ferroelectricity and ferromagnetism of the 3D nanocup heterostructure film at room temperature. 

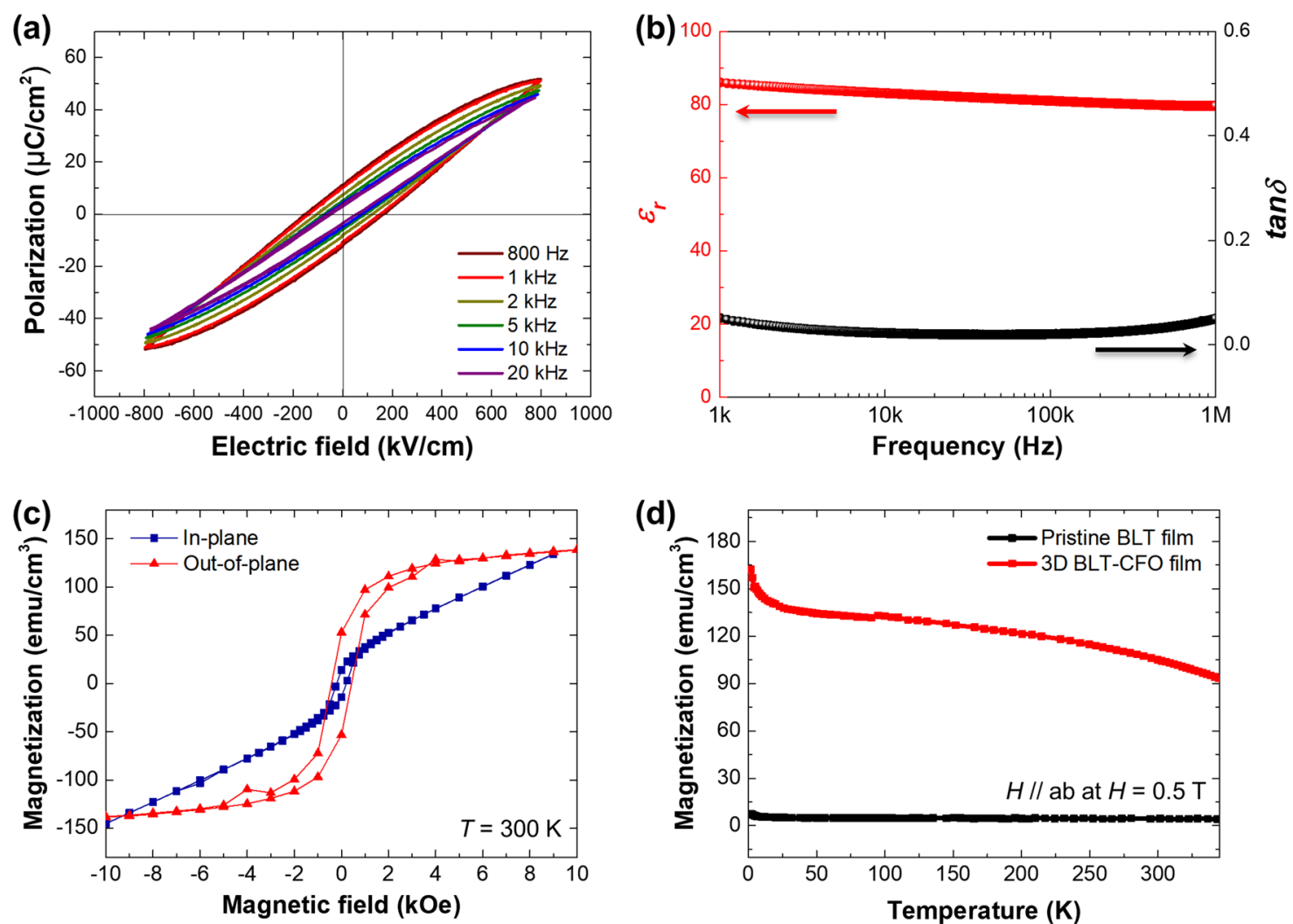

Fig. 4 Ferroelectric and ferromagnetic properties at room temperature. a $P-E$ loops for the 3D BLT-CFO nanocomposite films with thicknesses of $270 \mathrm{~nm}$ on the STO (110) substrate at various frequencies. b Dielectric constant and dielectric loss for the films with thicknesses of $270 \mathrm{~nm}$ on STO (001) substrate depending on the frequency. $\mathbf{c}$ In-plane and out-of-plane MH curves for the 3D BLT-CFO film on STO (001). d MT curves for the pristine BLT and 3D BLT-CFO film on STO (001) at $H=0.5 \mathrm{~T}$.

\section{Magnetoelectric coupling effect at room temperature}

To verify the ME coupling effect of the system, we performed magnetodielectric (MD) measurements at room temperature. Using MD measurements, we can analyze the variation in the dielectric constant $(\varepsilon)$ as a function of the magnetic field $(H)$. The ME coupling effect is assessed to conveniently quantify $\Delta \varepsilon$ (\%), according to the following equation ${ }^{44}$ :

$$
\Delta \varepsilon(\%)=[\{\varepsilon(H)-\varepsilon(0)\} / \varepsilon(0)] \times 100 .
$$

where $\varepsilon(H)$ is the dielectric constant under a static magnetic field $H$, and $\varepsilon(0)$ is that under a zero-bias field. To minimize the external resistivity, a sample for MD measurement was prepared, as schematically shown in Fig. 5a. The $\varepsilon(H)$ for the sample was measured by applying a magnetic field along the $c$-axis.

In heterostructure films, the MD values can be significantly affected by the magnetoresistance (MR) effect combined with the Maxwell-Wagner effect, which is a change in the resistance in the charge-depletion region due to a magnetic field ${ }^{45}$. Such a large MR effect makes it difficult to investigate the intrinsic ME coupling effect.
Hence, it is important to eliminate such an MR effect by investigating the dielectric loss and frequency dependence for identification of the intrinsic ME coupling effect. At a relatively high frequency $(800 \mathrm{kHz})$, symmetrical switching of the dielectric constant $(\Delta \varepsilon: 0.01 \%)$ was observed without any variation in the dielectric loss $(\tan \delta<0.1)$; the magnetic field was varied from -2 to $+2 \mathrm{~T}$ (Fig. 5b). Notably, reversible ME switching was consistently observed even during repeated tests (Fig. 5c). In addition, similar MD variations $(\Delta \varepsilon: 0.01 \%)$ were clearly observed at various frequencies, and the absence of leakage was confirmed, except for a slight fluctuation in the dielectric loss at $10 \mathrm{kHz}$ (Fig. 5d and Fig. S5, Supplementary Information). Furthermore, we investigated the MD effect for the pristine BLT and did not observe any MD variations, except for a continuous decrease in the dielectric constant over time (Fig. S6, Supplementary Information). As the continuous decrease in the dielectric constant was also observed for the 3D BLT-CFO film (insets of Fig. S5, Supplementary Information), the reduction in the dielectric constant in pristine BLT might be due to electric charge accumulation on the top and bottom electrodes caused by the AC electric field. Consequently, the 

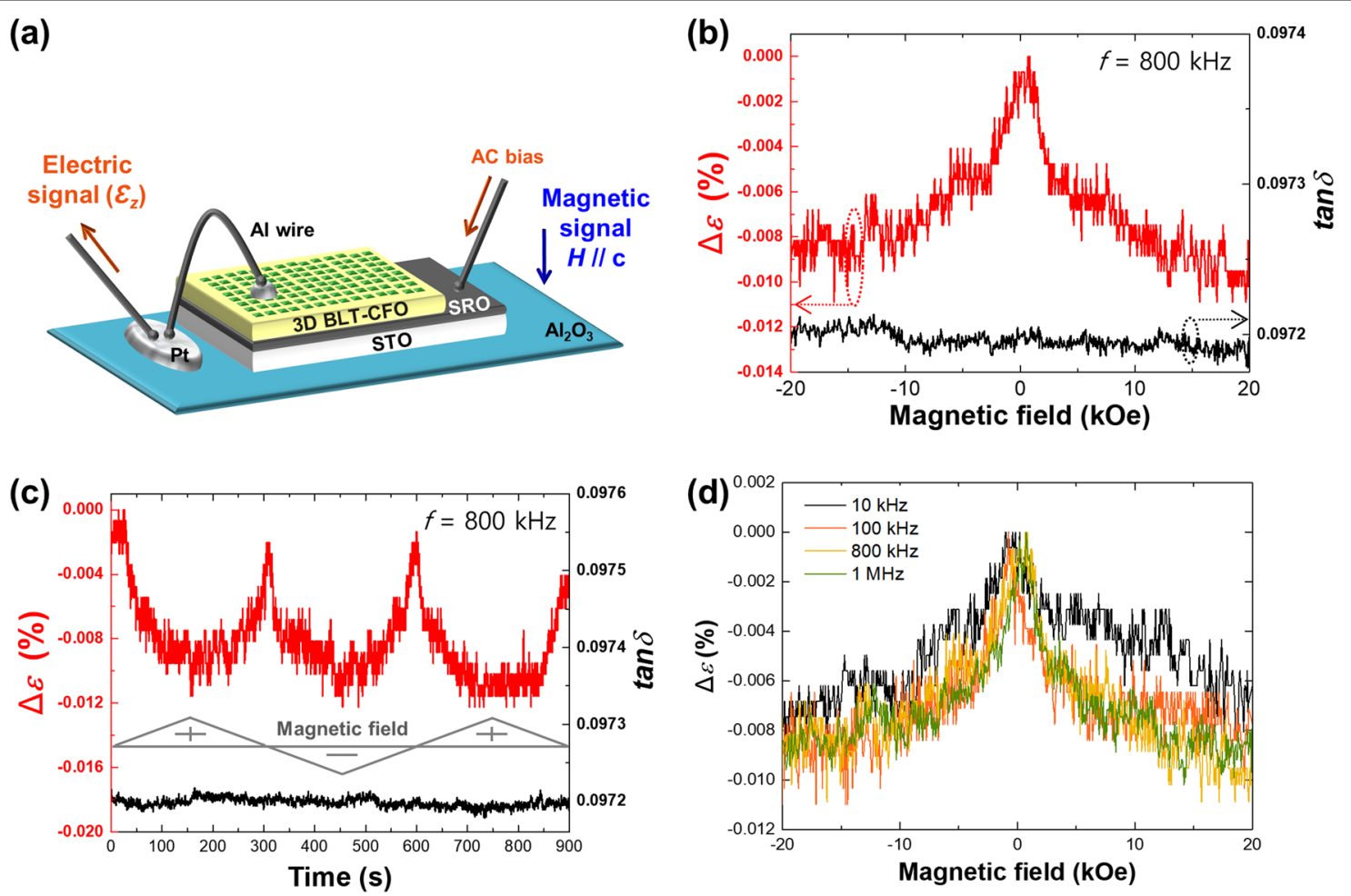

Fig. 5 Reversible magnetoelectric switching at room temperature. a Schematic illustration of the MD measurement system and sample configuration of the 3D BLT-CFO film on STO (001). b Magnetic field-dependent MD curve of the film at a frequency of $800 \mathrm{kHz}$. c Time-dependent MD curve of the film during the retention test from $-2 T$ to $2 \mathrm{~T}$. $\mathbf{d}$ Magnetic field-dependent MD curve of the film at various frequencies.

3D nanocup BLT-CFO film exhibited ME coupling at room temperature, which was not observed in BLT films. The magnetic field control of the ferroelectric behavior of the 3D nanocup film can be explained by strain-mediated ME coupling, as mostly observed in multiferroic heterostructure films. Hence, the magnetostriction generated on the ferromagnetic CFO nanocups by the magnetic field transformed the lattice of the ferroelectric BLT phase through their coherent interface, and then, the latticestrained BLT changed the MD values via piezoelectric properties. These results imply that the 3D nanocup film has an effective strain mediation between ferroelectric and ferromagnetic phases based on its excellent interfacial coherence.

In addition, based on the MD variation, which was proven from the MR artifacts combined with the Maxwell-Wagner effect, we calculated the magnetoelectric (ME) susceptibility $\left(\chi_{M E}\right)$ for the 3D nanocup heterostructure film using the following equation ${ }^{44}$ :

$$
\{\varepsilon(H)-\varepsilon(0)\} / \varepsilon(0)=\left(X_{M E} \cdot H\right) / E_{0}
$$

where the amplitude of the probing $\mathrm{AC}$ field $E_{O}$ is $37.0 \mathrm{KV} / \mathrm{cm}$. We obtained $\chi_{M E}=0.185 \mathrm{mV} / \mathrm{Oe} \cdot \mathrm{cm}$ for a magnetic field between 0 and $20 \mathrm{kOe}$. Although the degree of ME susceptibility is not as pronounced as that $(11.4 \mathrm{mV} / \mathrm{Oe} \cdot \mathrm{cm})$ of a polycrystalline $\mathrm{BiFeO}_{3} \mathrm{film}^{44}$, it is comparable to that $(0.16 \mathrm{mV} / \mathrm{Oe} \cdot \mathrm{cm})$ of typical multiferroic composites $\left(\mathrm{BaTiO}_{3}-\mathrm{CoFe}_{2} \mathrm{O}_{4}\right)^{46}$ for probing frequencies higher than their conductivity cutoff. Notably, reversible MD switching in our 3D nanocup film was reported for the first time. At the beginning of the MD measurement, the $\mathrm{MD}$ values have been reported without proof of the variation in the dielectric loss and frequency dependence, although the MR effect combined with the Maxwell-Wagner effect may be included. However, dielectric loss and frequency dependence should be verified to confirm genuine ME coupling because Catalan published a paper titled "Magnetocapacitance without magnetoelectric coupling" in $2006^{45}$. Recently, verification of reversible and reproducible MD variation has also been required for practical device applications. For a brief overview of the above, we summarized some reports that have the most reliable MD values via various verifications from past to present in Table $1^{47-51}$. Compared with the reported data in Table 1, we validated not only the intrinsic MD variation in which the MR artifacts combined with the Maxwell-Wagner effect were almost eliminated but also the reversible MD switching at room temperature. The reversible ME switching is noteworthy 
Table 1 The trend of MD measurements from past to present.

\begin{tabular}{|c|c|c|c|c|c|c|}
\hline Year & Materials & Temperature & Magnetic field & Frequency & Retention test & Ref. \\
\hline 2003 & $\mathrm{TbMnO}_{3}$ (Single crystal ceramic) & $12 \mathrm{~K}$ & 0 to $+9 \mathrm{~T}$ & $10 \mathrm{kHz}$ & - & 47 \\
\hline 2007 & $\mathrm{BiFeO}_{3}$ (Single crystal ceramic) & $250 \mathrm{~K}$ & 0 to $+9 \mathrm{~T}$ & $1 \mathrm{kHz}$ & - & 48 \\
\hline 2008 & La doped $\mathrm{BiFeO}_{3}$ (Single crystal film) & $300 \mathrm{~K}$ & 0 to $+1.2 \mathrm{~T}$ & $10 \mathrm{kHz}$ to $1 \mathrm{MHz}$ & - & 44 \\
\hline 2013 & $\mathrm{BiFeO}_{3}-\mathrm{CoFe}_{2} \mathrm{O}_{4}$ (Nanocomposite film) & $10 \mathrm{~K}$ & $-0.8 \mathrm{~T}$ to $0.8 \mathrm{~T}$ (Switchable) & $10 \mathrm{kHz}$ & - & 49 \\
\hline 2014 & Sm-doped $\mathrm{BiFeO}_{3}$ (Single crystal ceramic) & $300 \mathrm{~K}$ & 0 to $+1.5 \mathrm{~T}$ & $100 \mathrm{kHz}$ & - & 50 \\
\hline 2014 & (1-x) $\mathrm{BaTiO}_{3}-\mathrm{x} \mathrm{CoFe}_{2} \mathrm{O}_{4}$ (Composite ceramic) & $300 \mathrm{~K}$ & 0 to $7 \mathrm{~T}$ & $100 \mathrm{~Hz}$ to $1 \mathrm{MHz}$ & - & 46 \\
\hline 2016 & $\mathrm{LuFeO}_{3}$ (Nanocomposite film) & $300 \mathrm{~K}$ & 0 to $2 \mathrm{~T}$ & $10 \mathrm{kHz}$ to $1 \mathrm{MHz}$ & Reversible & 51 \\
\hline Our work & $\mathrm{Bi}_{3.25} \mathrm{La}_{0.75} \mathrm{Ti}_{3} \mathrm{O}_{12}-\mathrm{CoFe}_{2} \mathrm{O}_{4}$ (Nanocomposite film) & $300 \mathrm{~K}$ & $-2 \mathrm{~T}$ to $2 \mathrm{~T}$ (Switchable) & $10 \mathrm{kHz}$ to $1 \mathrm{MHz}$ & Reversible & \\
\hline
\end{tabular}

because it is difficult to investigate a genuine ME coupling effect owing to the relatively large MR artifacts and leakage issues in self-assembled nanocomposite films ${ }^{49}$. Therefore, our result may support the feasibility of ME devices because the reliable and sustainable ME effect is one of the fundamental requirements for practical devices.

How does reversible ME switching appear? As explained in the introduction, it is derived from the structural benefits of the 3D nanocup architecture. From the structural point of view, the 3D nanocup heterostructure film has excellent interfacial lattice coherence along both the in-plane and out-of-plane directions because BLT and CFO phases are self-assembled during PLD, similar to $0-3$ and 1-3 type films (Fig. S2, Supplementary Information). In addition, our nanocomposite film has a larger interfacial area than conventional 1-3 type films because the CFO nanoparticles inside the BLT film consist of aggregates of small nanoparticles, as shown in Fig. 2. Notably, the 3D nanocup architecture can overcome leakage through magnetic nanopillars, which is a limitation of 1-3 type films because the CFO nanocups are not in electrical contact with the substrate, as shown in Fig. S2c and d (Supplementary Information). Consequently, the 3D nanocup architecture enables overcoming the structural limitations of conventional heterostructure films (the bad dispersion of 0-3 type films, the substrate clamping effect of 2-2 type films, and the leakage problem of $1-3$ type films ${ }^{14}$ ).

\section{Conclusion}

In summary, we fabricated a 3D nanocup BLT-CFO film by inducing spontaneous nucleation and agglomeration of CFO during PLD. We demonstrated that the film had strong multiferroism without phase transformation of the pristine ferroelectric phase at room temperature, which, to the best of our knowledge, is the first report of its kind. In particular, the novel architecture of the $3 \mathrm{D}$ nanocup heterostructure film enables magnetically controlled reversible dielectric switching via overcoming the leakage issue, as well as the large interfacial area and excellent interfacial lattice coherence. We believe that the development of 3D nanocup heterostructure films facilitates the vitalization of a practical ME device in the near future.
Furthermore, considering the simplicity of our approach, fabrication of the innovative nanocup architecture can be widely applied for various complex oxides, ultimately leading to a variety of $3 \mathrm{D}$ nanocup-grafted heterostructure films for diverse multifunctional applications.

\section{Acknowledgements}

H.A. and H.J.H. contributed equally to this work. We thank K.S. and K.H.K. for discussions. This research was supported by the Creative Materials Discovery Program (2017M3D1A1040828), the Basic Science Research Program (2016R1D1A1B03931748), and a grant (NRF2013S1A2A2035468) through the National Research Foundation of Korea (NRF) funded by the Ministry of Education and the Ministry of Science and ICT and the National Research Foundation of Korea (NRF). B.K. acknowledges support from the National Research Foundation (NRF) Korea (No. 2018R1D1A1A02086051) and KISTI supercomputing center (Project No. KSC-2018-CRE-0079). This work was also supported by the KIST institutional program and a National Research Council of Science and Technology (NST) grant (No. CAP-16-01-KIST). K.-T.K. was supported by MPK (No. 2016K1A4A4A01922028).

\section{Author details}

${ }^{1}$ School of Materials Science and Engineering, Gwangju Institute of Science and Technology, Gwangju 61005, Republic of Korea. ${ }^{2}$ Center for Quantum Materials and Superconductivity (CQMS), Department of Physics,

Sungkyunkwan University, Suwon 16419, Republic of Korea. ${ }^{3}$ Department of Electrical Engineering, Gachon University, Seongnam 13120, Republic of Korea. ${ }^{4}$ Pohang Accelerator Laboratory (PAL), Pohang University of Science and Technology (POSTECH), Pohang 37673, Republic of Korea. ${ }^{5}$ Max Planck POSTECH/Hsinchu Center for Complex Phase Materials \& Department of Physics, POSTECH, Pohang 37673, Republic of Korea. ${ }^{6}$ Department of Physics, Kunsan National University, Gunsan 54151, Republic of Korea

Conflict of interest

The authors declare that they have no conflict of interest.

Publisher's note

Springer Nature remains neutral with regard to jurisdictional claims in published maps and institutional affiliations.

Supplementary information is available for this paper at https:/doi.org/ 10.1038/s41427-019-0172-4.

Received: 21 May 2019 Revised: 27 August 2019 Accepted: 24 September 2019.

Published online: 29 November 2019

\footnotetext{
References

1. Ramesh, R. \& Spaldin, N. A. Multiferroics: progress and prospects. Nat. Mater. $\mathbf{6}$, 21-27 (2007).
} 


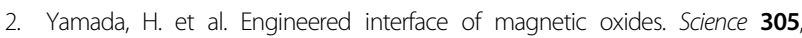
646-648 (2004)

3. Yang, H. et al. Vertical interface effect on the physical properties of selfassembled nanocomposite epitaxial films. Adv. Mater. 21, 3794-3798 (2009).

4. Mannhart, J. \& Schlom, D. G. Oxide interfaces - an opportunity for electronics. Science 327, 1607-1611 (2010).

5. Hwang, H. Y. et al. Emergent phenomena at oxide interfaces. Nat. Mater. 11 103-113 (2012).

6. Macmanus-Driscoll, J. L. et al. Strongly enhanced current densities in superconducting coated conductors of $\mathrm{YBa}_{2} \mathrm{Cu}_{3} \mathrm{O}_{7-x}+\mathrm{BaZrO}_{3}$. Nat. Mater. 3, 439-443 (2004).

7. Lee, S. et al. Template engineering of $\mathrm{Co}-$ doped $\mathrm{BaFe}_{2} \mathrm{As}_{2}$ single-crystal thin films. Nat. Mater. 9, 397-402 (2010).

8. Zheng, $\mathrm{H}$. et al. Multiferroic $\mathrm{BaTiO}_{3}-\mathrm{CoFe}_{2} \mathrm{O}_{4}$ nanostructures. Science $\mathbf{3 0 3}$ 661-663 (2004).

9. Harrington, S. A. et al. Thick lead-free ferroelectric films with high Curie temperatures through nanocomposite-induced strain. Nat. Nanotechnol. $\mathbf{6}$, 491-495 (2011).

10. Zhang, W. et al. Strain relaxation and enhanced perpendicular magnetic anisotropy in $\mathrm{BiFeO}_{3}: \mathrm{COFe}_{2} \mathrm{O}_{4}$ vertically aligned nanocomposite thin films. Appl. Phys. Lett. 104, 062402 (2014).

11. Chen, A. et al. Tunable low-field magnetoresistance in $\left(\mathrm{La}_{0.7} \mathrm{Sr}_{0.3} \mathrm{MnO}_{3}\right)_{0.5}$ : $(\mathrm{ZnO})_{0.5}$ self-assembled vertically aligned nanocomposite thin films. Adv. Funct. Mater. 21, 2423-2429 (2011).

12. Lee, S. et al. Novel electroforming-free nanoscaffold memristor with very high uniformity, tunability, and density. Adv. Mater. 26, 6284-6289 (2014).

13. MaCmanus-Driscoll, J. L. et al. Strain control and spontaneous phase ordering in vertical nanocomposite heteroepitaxial thin films. Nat. Mater. 7, 314-320 (2008).

14. $\mathrm{Ma}, \mathrm{J}$., Hu, J., Li, Z. \& Nan, C. W. Recent progress in multiferroic magnetoelectric composites: from bulk to thin films. Adv. Mater. 23, 1062-1087 (2011).

15. Hu, J. M., Chen, L. Q. \& Nan, C. W. Multiferroic heterostructures integrating ferroelectric and magnetic materials. Adv. Mater. 28, 15-39 (2016).

16. Rani, A., Kolte, J. \& Gopalan, P. Investigation on the structural, multiferroic and magnetoelectric properties of $\mathrm{BaTi}_{1-x} \mathrm{Ni}_{x} \mathrm{O}_{3}$. Ceram. Int. 45, 5312-5320 (2019).

17. Trukhanov, S. et al. Preparation and investigation of structure, magnetic and dielectric properties of $\left(\mathrm{BaFe}_{11.9} \mathrm{Al}_{0.1} \mathrm{O}_{19}\right)_{1-x}-\left(\mathrm{BaTiO}_{3}\right)_{x}$ bicomponent ceramics. Ceram. Int. 44, 21295-21302 (2018).

18. Kitagawa, Y. et al. Low-field magnetoelectric effect at room temperature. Nat. Mater. 9, 797 (2010).

19. Chun, S. H. et al. Electric field control of nonvolatile four-state magnetization at room temperature. Phys. Rev. Lett. 108, 177201 (2012).

20. Zhang, W., Ramesh, R., MacManus-Driscoll, J. L. \& Wang, H. Multifunctional, selfassembled oxide nanocomposite thin films and devices. MRS Bull. 40, 736-745 (2015).

21. Wang, Y., Hu, J., Lin, Y. \& Nan, C.-W. Multiferroic magnetoelectric composite nanostructures. NPG Asia Mater. 2, 61-68 (2010).

22. Alexe, M. et al. Ferroelectric switching in multiferroic magnetite $\left(\mathrm{Fe}_{3} \mathrm{O}_{4}\right)$ thin films. Adv. Mater. 21, 4452-4455 (2009).

23. Vaz, C. A. F., Hoffman, J., Ahn, C. H. \& Ramesh, R. Magnetoelectric coupling effects in multiferroic complex oxide composite structures. Adv. Mater. 22, 2900-2918 (2010).

24. Imai, A. et al. Epitaxial $\mathrm{Bi}_{5} \mathrm{TT}_{3} \mathrm{FeO}_{15}-\mathrm{CoFe}_{2} \mathrm{O}_{4}$ pillar-matrix multiferroic nanostructures. ACS Nano 7, 11079-11086 (2013).

25. Li, Y. et al. Magnetoelectric quasi-(0-3) nanocomposite heterostructures. Nat. Commun. 6, 6680 (2015)

26. Kim, D. H., Aimon, N. M., Sun, X. \& Ross, C. A. Compositionally modulated magnetic epitaxial spinel/perovskite nanocomposite thin films. Adv. Funct. Mater. 24, 2334-2342 (2014).
27. Ojha, S., Nunes, W. C., Aimon, N. M. \& Ross, C. A. Magnetostatic interactions in self-assembled $\mathrm{CO}_{x} \mathrm{Ni}_{1-x} \mathrm{Fe}_{2} \mathrm{O}_{4} / \mathrm{BiFeO}_{3}$ multiferroic nanocomposites. ACS Nano 10, 7657 (2016)

28. Aimon, N. M., Choi, H. K., Sun, X. Y., Kim, D. H. \& Ross, C. A. Templated selfassembly of functional oxide nanocomposites. Adv. Mater. 26, 3063-3067 (2014).

29. Tian, $\mathrm{G}$. et al. Magnetoelectric coupling in well-ordered epitaxial $\mathrm{BiFeO}_{3} /$ $\mathrm{CoFe}_{2} \mathrm{O}_{4} / \mathrm{SrRuO}_{3}$ heterostructured nanodot array. ACS Nano 10, 1025-1032 (2016).

30. Kresse, G. \& Hafner, J. Ab initio molecular dynamics for liquid metals. Phys. Rev B 47, 558-561 (1993).

31. Kresse, G. \& Furthmüller, J. Efficient iterative schemes for ab initio total-energy calculations using a plane-wave basis set. Phys. Rev. B 54, 11169-11186 (1996).

32. An, H. et al. Large enhancement of the photovoltaic effect in ferroelectric complex oxides through bandgap reduction. Sci. Rep. 6, 28313 (2016).

33. Han, J. Y. \& Bark, C. W. Influence of transition metal doping $(X=\mathrm{Co}$, Fe) on structural, optical properties of ferroelectric $\mathrm{Bi}_{3.25} \mathrm{La}_{0.75} \mathrm{X}_{1} \mathrm{Ti}_{2} \mathrm{O}_{12}$. Nano Convergence 2, 1-5 (2015).

34. Song, M. G., Han, J. Y. \& Bark, C. W. Effects of doping ratio of cobalt and iron on the structure and optical properties of $\mathrm{Bi}_{3.25} \mathrm{La}_{0.75} \mathrm{Fe}_{x} \mathrm{CO}_{1-x} \mathrm{Ti}_{2} \mathrm{O}_{12}(x=0,0.25$, $0.5,0.75,1$ ). J. Nanosci. Nanotechnol. 15, 7841-7844 (2015).

35. Park, B. H. et al. Lanthanum-substituted bismuth titanate for use in non-volatile memories. Nature 401, 682-684 (1999).

36. Neagu, D., Tsekouras, G., Miller, D. N., Ménard, H. \& Irvine, J. T. S. In situ growth of nanoparticles through control of non-stoichiometry. Nat. Chem. 5, 916-923 (2013).

37. Sun, Y.-F. et al. New opportunity for in situ exsolution of metallic nanoparticles on perovskite parent. Nano Lett. 16, 5303-5309 (2016).

38. Kwon, O. et al. Exsolution trends and co-segregation aspects of self-grown catalyst nanoparticles in perovskites. Nat. Commun. 8, 15967 (2017).

39. Ohring, M. Materials Science of Thin Films (pp. 429-439. Academic Press, San Diego, CA, USA, 2001).

40. Zheng, H. M. et al. Self-Assembled growth of $\mathrm{BiFeO}_{3}-\mathrm{CoFe}_{2} \mathrm{O}_{4}$ nanostructures. Adv. Mater. 18, 2747-2752 (2006).

41. Kashchiev, D. Nucleation: Basic Theory with Applications (Butterworth-Heinemann, Oxford, UK, 2000).

42. Lee, H. N. \& Hesse, D. Anisotropic ferroelectric properties of epitaxially twinned $\mathrm{Bi}_{3.25} \mathrm{La}_{0.75} \mathrm{TT}_{3} \mathrm{O}_{12}$ thin films grown with three different orientations. Appl. Phys. Lett. 80, 1040-1042 (2002).

43. Choi, W. S. et al. Wide bandgap tunability in complex transition metal oxides by site-specific substitution. Nat. Commun. 3, 689 (2012).

44. Jang, H. M., Park, J. H., Ryu, S. W. \& Shannigrahi, S. R. Magnetoelectric coupling susceptibility from magnetodielectric effect. Appl. Phys. Lett. 93, 252904 (2008).

45. Catalan, G. Magnetocapacitance without magnetoelectric coupling. Appl. Phys. Lett. 88, 102902 (2006)

46. Shen, $\mathrm{Y}$. et al. The enhanced magnetodielectric interaction of $(1-\mathrm{x})$ $\mathrm{BaTiO}_{3-x} \mathrm{CoFe}_{2} \mathrm{O}_{4}$ multiferroic composites. J. Mater. Chem. C 2, 2545 (2014).

47. Kimura, $\mathrm{T}$. et al. Magnetic control of ferroelectric polarization. Nature $\mathbf{4 2 6}$, 55-58 (2003).

48. Kamba, S. et al. Infrared and terahertz studies of polar phonons and magnetodielectric effect in multiferroic $\mathrm{BiFeO}_{3}$ ceramics. Phys. Rev. B 75, 024403 (2007).

49. Stratulat, S. M. et al. Nucleation-induced self-assembly of multiferroic $\mathrm{BiFeO}_{3}-\mathrm{CoFe}_{2} \mathrm{O}_{4}$ nanocomposites. Nano. Lett. 13, 3884-3889 (2013).

50. Mukherjee, A. et al. Giant magnetodielectric and enhanced multiferroic properties of Sm doped bismuth ferrite nanoparticles. J. Mater. Chem. C 2, 5885-5891 (2014)

51. Song, S. et al. Implementing room-temperature multiferroism by exploiting hexagonal-orthorhombic morphotropic phase coexistence in $\mathrm{LuFeO}_{3}$ thin films. Adv. Mater. 28, 7430 (2016). 